\title{
DIAGNÓSTICO DOS RESÍDUOS SÓLIDOS DA LAVRA DE ROCHA ORNAMENTAL DA PEDREIRA GRANORTE, PARELHAS-RN
}

\author{
R.A. PEREIRA ${ }^{1}$, J.C. PONTES \\ Instituto Federal de Educação, Ciência e Tecnologia do Rio Grande de Norte \\ ranieri.engminas@gmail.com ${ }^{1}$
}

Artigo submetido em 25/11/2019 e aceito em 11/12/2019

DOI: $10.15628 /$ holos.2019.9204

\section{RESUMO}

Este trabalho versa sobre o diagnóstico ambiental e de processos com vistas à implementação da ferramenta de gestão ambiental de Produção Mais Limpa nos anos de 2016 e 2017, na pedreira da empresa Granorte Mineração de Mármores e Granitos do Rio Grande do Norte, localizada na zona rural de Parelhas-RN. O objetivo do trabalho foi diagnosticar a situação de geração de resíduos sólidos nas frentes de lavra. 0 diagnóstico para implementação de técnicas de Produção Mais Limpa para lavra de rocha ornamental seguiu a metodologia do Centro Nacional de Tecnologias Limpas do SENAI. As atividades programadas foram desenvolvidas $e$ organizadas envolvendo pesquisa bibliográfica, e levantamento de dados do processo de lavra, visita de campo e trabalhos de laboratório. A pesquisa bibliográfica foi realizada através de consulta em banco
\end{abstract}

de dados, revistas, periódicos e livros. Foi realizada a coleta de duas amostras de resíduos para análises químicas do tipo Fluorescência de Raio-X. Essas análises identificaram pequenos traços de elemento de terras raras, como também a presença de óxido de silício em percentuais que podem ser aproveitados. A quantidade de rocha e solo movimentados no período do estudo foi de $17.487,00$ toneladas, sendo $11.768,75$ descartados para pilha de resíduos, e 5.718,25 toneladas como produto na forma de blocos. 0 estudo identificou que 99,97 \% em massa dos resíduos sólidos gerados nas frentes de lavra são de fragmentos de rochas. Entretanto, os resultados também demonstraram que técnicas de Produção Mais Limpa como modificações no processo e a aplicação de boas práticas operacionais.

PALAVRAS-CHAVE: Diagnóstico, Produção Mais Limpa, Rocha Ornamental, Lavra, Resíduos Sólidos.

\section{DIAGNOSIS OF THE SOLID WASTE OF THE ORNAMENTAL ROCK WASH OF PEDREIRA GRANORTE, PARELHAS-RN}

\begin{abstract}
This paper deals with the environmental and process diagnosis with a view to the implementation of the Cleaner Production environmental management tool in 2016 and 2017, in the quarry of the company Granorte Mining of Marbles and Granites of Rio Grande do Norte, located in the area rural area of Parelhas-RN. The objective of this work was to diagnose the situation of solid waste generation in the mining fronts. The diagnosis for implementation of Cleaner Production techniques for ornamental rock mining followed the methodology of SENAI's National Center for Clean Technologies. The programmed activities were developed and organized involving bibliographic research, data mining of the mining process, field visit and laboratory work. The bibliographic search was performed through consultation
\end{abstract}

in databases, magazines, journals and books. Two residue samples were collected for chemical analysis of the X-ray Fluorescence type. These analyzes identified small traces of rare earth elements, as well as the presence of silicon oxide in percentages that can be exploited. The amount of rock and soil handled during the study period was $17,487.00$ tons, of which $11,768.75$ tons were disposed of as waste piles, and 5,718.25 tons as product in the form of blocks. The study identified that $99.97 \%$ by mass of solid residues generated on the mining fronts are from rock fragments. However, the results also demonstrated that Cleaner Production techniques such as process modifications and the application of good operating practices.

KEYWORDS: Diagnosis, Cleaner production, Ornamental Rock, Mining, Solid waste. 


\section{INTRODUÇÃO}

O interesse do homem pelos produtos da mineração vem desde a Antiguidade, vendo, nestes, qualidades estéticas ou procurando neles propriedades físico-mecânicas (CURI, 2014).

O setor de mineração figura como uma atividade econômica de grande importância para o Estado do Rio Grande do Norte. A área de estudo está localizada na comunidade quilombola Boa Vista dos Negros, município de Parelhas-RN. A lavra de rocha ornamental é tão significativa para o município que tem gerado emprego e renda, podendo figurar como um arranjo produtivo local. Na pauta de Exportação de mármores e granitos em estado bruto ou desbastados por unidades da federação produtora, no ano de 2016 e no acumulado de janeiro a maio de 2017, ocupa a 5á posição no cenário nacional e o $3^{3}$ da região nordeste (BRASIL, 2017). Segundo dados do Sistema de Informações Geográficas da Mineração (SIGMINE) da Agência Nacional de Mineração em fevereiro de 2017, o município possuía cerca de 158 processos minerários, sendo que 21 destes processos se encontram em fase de lavra e apenas $01(\mathrm{um})$, com portaria de lavra autorizado pelo Ministério de Minas e Energia para rocha ornamental. Todavia, é sabido que existem inúmeras operações de lavra de rocha ornamental autorizadas de forma provisória, com período de um ano através do documento de Guia de Utilização e Licenciamento Ambiental. Estas autorizações provisórias demonstram o grande entrave processual junto a autarquia federal que regula o setor mineral e que indiretamente contribuem para que a atividade de mineração seja realizada de forma sazonal.

A sustentabilidade passa pela concepção de que uma atividade que causa impactos ambientais como é o caso da mineração, seja desenvolvida de maneira que assegure as gerações futuras a mesma disponibilidade de recursos naturais daquela geração na qual o empreendimento operou.

\section{REVISÃO BIBLIOGRÁFICA}

Os recursos naturais provenientes da mineração não são renováveis, e por se tratar de uma atividade humana, podem degradar o meio ambiente significativamente. De acordo com (VENTURI, 2006) recurso natural pode ser definido como qualquer elemento ou aspecto da natureza que esteja em demanda, seja passível de uso ou esteja sendo usado pelo Homem, direta ou indiretamente, como forma de satisfação de suas necessidades físicas e culturais em determinado tempo e espaço.

Sabe-se que a rocha ornamental é um recurso natural não renovável. Portanto, a apropriação desse recurso de forma predatória pode levar a sua escassez e consequentemente os resultados dos impactos ambientais ocasionados pela atividade de extração serão potencializados principalmente no solo devido à disposição inadequada de resíduos gerados nas operações de lavra.

Devido à rocha ornamental ter um padrão estético preestabelecido para sua aplicação, as operações unitárias para extração desse recurso se tornam complexas, devido ao seu aproveitamento na lavra. As propriedades intrínsecas das rochas compreendem parâmetros físicos, físico-mecânicos e químico mineralógicos. São determinados mediante análises e ensaios 
tecnológicos, executados sob rigorosos procedimentos padronizados, norteados por instruções normatizadoras, e constituem a caracterização tecnológica das rochas (MENEZES, 2005).

Não atendo-se apenas as análises e ensaios tecnológicos para determinação de parâmetros físicos, tais como; resistência ao impacto e desgaste, a lavra da rocha ornamental são limitadas as estruturas geológicas, o que pode interferir diretamente na produção da mina e geração de resíduos. Elementos com falhas e fraturas podem causar sérios problemas para a operação da mina e consequentemente impactos ambientais negativos.

A medida que se caracteriza as propriedades químicas e físicas da rocha, bem como suas estruturas geológicas, chega-se à conclusão de como este maciço rochoso pode se comportar após os cortes que serão realizados na rocha nas atividades de lavra. Consegue-se determinar as zonas de alívio de tensão e por fim as melhores direções de avanço da lavra para que se tenha uma Produção Mais Limpa.

De acordo com Campos et al. (2009, p. 12), os resíduos grosseiros de pedreiras são compostos de blocos de tamanhos irregulares, blocos com imperfeições ocasionados no manejo, blocos com mudanças de padrão de cores e lascas de rochas.

Diante de um cenário com grande produção de resíduos, entende-se que a Produção Mais Limpa pode ser uma estratégia técnica, econômica e ambiental integrada aos processos e produtos, a fim de aumentar a eficiência no uso de matérias-primas, água e energia, através da não geração, minimização ou reciclagem dos resíduos e emissões geradas, com benefícios ambientais, de saúde ocupacional e econômicos (SENAI-RS, 2003a).

\section{METODOLOGIA}

O estudo dos problemas ambientais causados pela produção de resíduos sólidos de lavras de rochas ornamentais teve por início com o levantamento de dados secundários em consultas bibliográficas em bancos de artigos, teses e dissertações do domínio público e periódicos, bem como em anais de congressos e simpósios no tema de rochas ornamentais e produção mais limpa.

Os dados primários foram obtidos em campo, através do preenchimento de formulário de entrevistas feitas com funcionários e proprietário da empresa, em três visitas a Pedreira na cidade de Parelhas, região Seridó, estado do Rio Grande do Norte, entre os anos de 2015 e 2016.

O presente trabalho se constitui pela abordagem qualitativa e quantitativa e de caráter exploratório e descritivo, pois se pretende descrever o processo de lavra de rochas ornamentais, e, através da coleta de dados, avaliar a geração de resíduos da atividade (SAMPIERI, COLLADO, LUCIO, 2006), utilizando para isso bibliografias e estudo de caso. O estudo de caso foi pautado no diagnóstico de resíduos e teve por base o método do Centro Nacional de Tecnologias Limpas (CNTL/SENAI-RS) seguindo os passos para implementação (Figura 01). Com a seguinte sequência: acompanhamento das atividades, investigação dos poluentes disseminados no meio ambiente motivados pela atividade de lavra por meio de observação in loco e análises químicas, sensibilização da gerência, identificação de barreiras e soluções para, formação do ecotime, elaboração do diagnóstico para definição do processo a ser estudado para a implementação da Produção Mais 
Limpa na lavra de rocha ornamental, análise da geologia estrutural das frentes de lavra com vistas a redução de resíduos, e monitoramento das oportunidades de técnicas de Produção Mais Limpa para a operação de lavra.

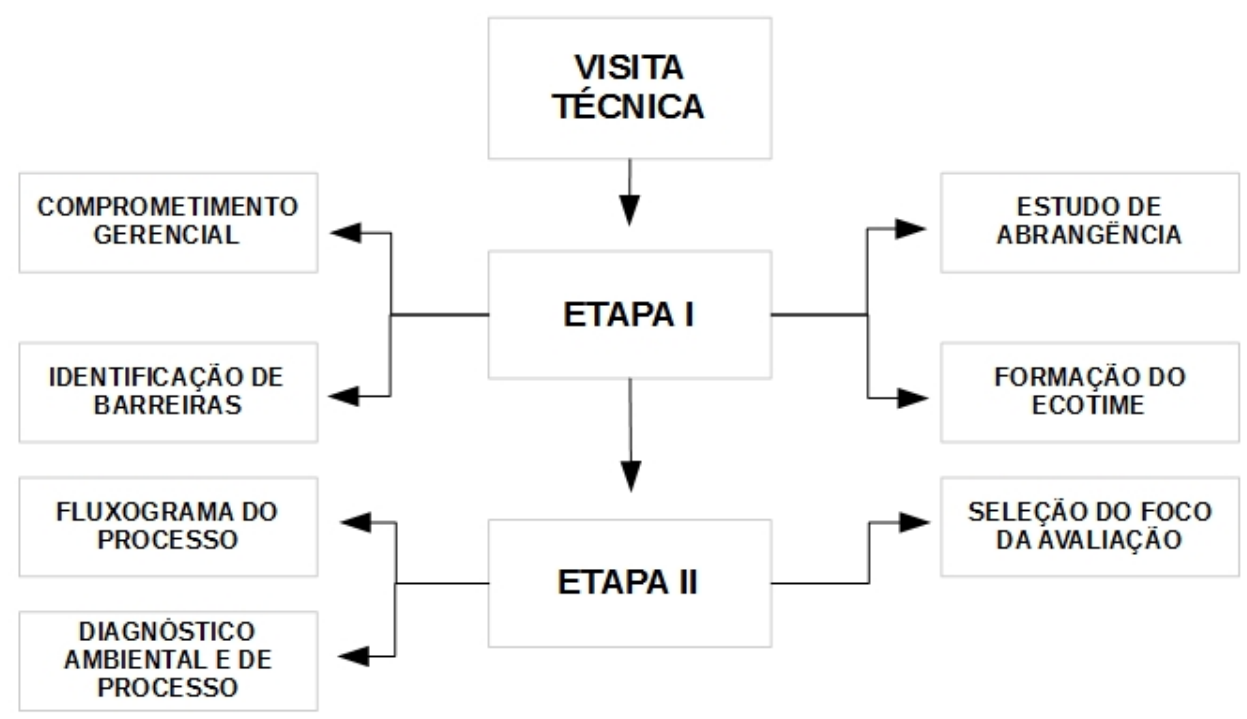

Figura 1: Fluxograma da metodologia adotada no trabalho.

\section{RESULTADOS E DISCUSSÃO}

\subsection{Equipamentos}

Os equipamentos utilizados no processo em sua maioria são movidos com motores de combustão a óleo diesel. Apenas a máquina de fio cortadora que possui motor elétrico, mas o fornecimento desta energia tem origem no gerador que utiliza um motor a diesel.

Tabela 1: Equipamentos com os dados do formulário do diagnóstico.

\begin{tabular}{c|c|c|c|c|c|c}
\hline $\mathrm{N}^{\circ}$ & Equipamento & Quantidade & $\begin{array}{c}\text { Capacidade } \\
\text { Nominal }\end{array}$ & Operação & Ano & Manutenção \\
\hline 1 & $\begin{array}{c}\text { Escavadeira } \\
\text { Volvo 220 DL }\end{array}$ & 1 & $1.800 \mathrm{rpm}$ & Lavra & 2014 & $02 / 2017$ \\
\hline 2 & $\begin{array}{c}\text { Pá Carregadeira } \\
\text { Volvo L120F }\end{array}$ & 1 & $1.700 \mathrm{rpm}$ & Lavra & 2015 & $04 / 2017$ \\
\hline 3 & Caminhão Pipa & 1 & $5.000 \mathrm{Its}$. & Lavra & 2012 & $03 / 2017$ \\
\hline 4 & $\begin{array}{c}\text { Compressor } \\
\text { Atlas Copco }\end{array}$ & 2 & $400 \mathrm{PCM}$ & Lavra & 2014 & $02 / 2017$ \\
\hline 5 & Gerador & 1 & $75 \mathrm{kva}$ & Lavra & 2010 & $04 / 2017$ \\
\hline
\end{tabular}




\begin{tabular}{c|c|c|c|c|c|c}
\hline 6 & $\begin{array}{c}\text { Martelo Fundo } \\
\text { Furo (DTH) }\end{array}$ & 1 & Lavra & 2015 & $03 / 2017$ \\
\hline 7 & Martelo Manual & 2 & & Lavra & 2015 & $02 / 2017$ \\
\hline 8 & $\begin{array}{c}\text { Máquina } \\
\text { Cortadora }\end{array}$ & 1 & Lavra & 2014 & $02 / 2017$ \\
\hline 9 & $\begin{array}{c}\text { Perfuratriz sobre } \\
\text { trilho }\end{array}$ & 1 & Lavra & 2015 & $03 / 2017$ \\
\hline
\end{tabular}

\subsection{Consumo de água}

O consumo de água em média é de $5.800 \mathrm{I} /$ dia ou 1.670,4 $\mathrm{m}^{3} / \mathrm{ano}$, foi considerado alto e pode ser minimizado com o controle da operação de perfuração e corte. $O$ aspecto de consumo de água pode ocasionar os impactos de consumo de recursos naturais e redução da emissão de aerodispersoides. Sendo o encarregado e operadores da máquina de fio e perfuratriz, os responsáveis pela implementação desta medida.

Tabela 2: Consumo de água.

\begin{tabular}{c|c|c|}
\hline Operação & Consumo $\mathrm{m}^{3} / \mathrm{mês}$ & $\begin{array}{c}\text { Consumo } \\
\mathrm{m}^{3} / \text { ano }\end{array}$ \\
\hline Perfuração & 48 & 576 \\
Corte & 91,2 & $1.094,40$ \\
Total & 139,2 & $1.670,40$ \\
\hline
\end{tabular}

A operação de perfuração consome em média $48 \mathrm{~m}^{3} / \mathrm{mês}$, o que representa $34,48 \%$ do consumo, contra $91,2 \mathrm{~m}^{3} /$ mês da máquina de fio diamantado que representa $65,52 \%$ do consumo total de água no processo de lavra.

\subsection{Consumo de combustível e emissões}

Foram identificados em praticamente todas as etapas processo que há o aspecto ambiental de emissão de gases provenientes da queima de combustíveis, isso se dá devido ao grau de mecanização da operação de lavra e pela escolha da matriz energética do empreendimento. Em um ano, que correspondeu ao período de monitoramento para elaboração deste diagnóstico, foi catalogado um consumo médio de óleo diesel por equipamento e o somatório foi de 119.000,0 litros. O fator de emissão de CO2 para equipamentos pesados a diesel, de acordo com o método Bottom-up é de $3140 \mathrm{~g} / \mathrm{kg}$ de combustível. A densidade do diesel 5500 é de $850 \mathrm{~kg} / \mathrm{m}^{3}$. Estima-se que por mês, conforme o consumo levantado, esta mina produza emissões de $\mathrm{CO} 2$ de cerca de 7,95 toneladas, o que totalizou no ano do estudo de 95,47 toneladas. 
Tabela 3: Consumo de combustível.

\begin{tabular}{|c|c|c|c|}
\hline Equipamento & $\begin{array}{c}\text { Consumo } \\
\text { mensal } \\
\left(\mathrm{m}^{3}\right)\end{array}$ & $\begin{array}{c}\text { Consumo } \\
\text { anual }\left(\mathrm{m}^{3}\right)\end{array}$ & $\begin{array}{c}\mathrm{CO}_{2} \text { anual } \\
\text { (ton.) }\end{array}$ \\
\hline Escavadeira & 2,88 & 34,56 & 92,24 \\
Pá Carregadeira & 2,40 & 28,80 & 76,86 \\
Caminhão & 0,55 & 6,60 & 17,61 \\
Compressores & 2,16 & 25,92 & 69,18 \\
Gerador & 1,92 & 23,04 & 61,49 \\
Total & 9,91 & 118,92 & 317,38 \\
& & & \\
\hline
\end{tabular}

\subsection{Consumo de recurso mineral e resíduos gerados}

O principal insumo que ao ser modificado pode se transformar em produto ou resíduo é a rocha pegmatítica. Para isto foram utilizados os dados de relatórios de produção da atividade de lavra para quantificar o produto. No tocante aos resíduos de rochas ou solos, estes foram quantificados mediante observação nas frentes de produção realizadas através de medições comprimentos, largura e alturas dos blocos e fragmentos desperdiçados, desta maneira pôde-se chegar a valores aproximados do volume de resíduos de rochas. Quanto ao resíduo sólido do tipo plástico, foram quantificados os consumos mensais de argamassa expansiva, cada saco possui $5 \mathrm{~kg}$ do produto para o corte da rocha através do processo de esquadrejamento. De posse do conhecimento da demanda de argamassa, foi identificado o número de embalagens que por mês teve uma massa em média de $3,75 \mathrm{~kg}$, o que totalizou $45 \mathrm{~kg}$ no período de um ano. Quanto a sucata ferrosa, originada de quebras de equipamentos e peças foram quantificadas mediante a coleta por empresa especializada que realiza a coleta e teve uma massa de 3,0 toneladas.

No tocante ao ponto mais crítico da pesquisa, que está relacionado a geração de resíduos sólidos e em especial aqueles provenientes das frentes de lavra, no ano de 2016 foram gerados aproximadamente em resíduos $11.771,79$ toneladas, sendo $45 \mathrm{~kg}$ de embalagem plástica, 3 toneladas/ano de sucata ferrosa e 11.768,75 toneladas de fragmentos de rochas e solos (Figura 07).

O resíduo sólido do tipo fragmentos de rochas e solos representaram 99,97\% do percentual em massa dos resíduos produzidos na pedreira Granorte. Com base nos dados de entrada e saída para produção de blocos foi identificado um rendimento de aproximadamente $32,7 \%$, confirmando dados da literatura quando ao alto índice de desperdício para a mineração de rocha ornamental.

\subsection{Quantificação dos resíduos gerados por etapas do processo de lavra}

Nesta seção houve necessidade de fazer o acompanhamento da atividade com o controle e anotação de dados de produção anual (Tabela 4).

Tabela 4: Geração de resíduos de 2016/2017 em toneladas por etapas do processo. 


\begin{tabular}{|l|c|c|}
\hline \multicolumn{1}{|c|}{ Insumo } & Entrada & Saída \\
\hline Decapeamento/Limpeza & 17487 & 13367,94 \\
\hline Perfuração & 13367,94 & 13014,88 \\
\hline Corte & 13014,88 & 9955,01 \\
\hline Tombamento & 9955,01 & 7483,58 \\
\hline Desdobramento & 7483,85 & 5718,25 \\
\hline Total & & 5718,25 \\
\hline
\end{tabular}

Nisto foi identificado que $35 \%$ do recursos mineral é desperdiçado na forma de fragmentos de rocha, $26 \%$ tem origem pelo corte da rocha e fraturas pré existentes, $21 \%$ pela operação de tombamento, $15 \%$ no desdobramento $3 \%$ na operação de perfuração.

\subsection{Monitoramento de oportunidades para implementação da Produção Mais Limpa}

Sabe-se que o foco da Produção Mais Limpa está na prevenção, e para que se alcance este objetivo deve-se traçar metas que busquem a minimização ou eliminação de resíduos e emissões. Portanto, para este estudo buscou-se metas mais ambiciosas, as que se distanciam das técnicas de fim de tubo. Com base nos dados do fluxograma qualitativo e do formulário com informações quantitativas, foram identificadas as oportunidades na sequência por ordem de importância, considerando os maiores volumes de perdas como: decapeamento/limpeza, corte e tombamento, desdobramento, perfuração, como também operações de manutenção de equipamentos, controle de emissões, consumo de água e embalagens.

O tipo de resíduo sólido que apresentou maior quantidade absoluta e relativa foi o de mineração do tipo fragmentos de rochas. Resíduos de mineração na forma de blocos e fragmentos graúdos pertencem a classe II B por serem inertes. Entretanto, a preocupação está no volume que tem sido gerado e disposto sobre o solo. Nesta etapa a fonte de maior geração de resíduo identificada é a de decapeamento e limpeza. É sabido que estruturas geológicas e o grau de intemperismo em que as rochas se encontram nas suas camadas superficiais, podem influenciar diretamente na qualidade do material rochoso e nos aspectos estéticos e físicos, tornando muitas vezes a operação de decapeamento e limpeza essencial. No entanto, pode-se racionalizar esta operação, realizando-a apenas quando necessária, o que caracteriza uma mudança no processo ou até mesmo boas práticas operacionais. Outra oportunidade para implementação da Produção Mais Limpa foi a de reciclagem interna do resíduo proveniente do decapeamento e limpeza, cortes, tombamento e desdobramento. Na literatura existem estudos que indicam a reutilização de resíduos sólidos de rochas ornamentais em diversos setores industriais. Para isto, realizou-se análises químicas para determinação de percentuais de óxidos visando caracterizar o resíduo com o objetivo de torná-lo produto.

Quanto a situação de resíduos gerados na empresa, recomenda-se que sejam realizados mais análises químicas e físicas a fim de complementar a caracterização tecnológica iniciada neste estudo, e assim realizar um planejamento para implementar a reciclagem interna. Entretanto, 
diante dos dados da análise (Figura 02), vislumbra-se diversas aplicações, por exemplo como agregados na construção civil e cerâmica. No entanto, percebe-se a presença de alguns óxidos de metais raros que podem ter aplicações nobres e outros que merecem uma atenção e estudos mais aprofundados devido a possível toxidade.

\begin{tabular}{|l|r|}
\hline \multicolumn{2}{|c|}{ Amostra A - Retido \#200 } \\
\hline Oxido & Percentual \\
\hline $\mathrm{SiO}_{2}$ & 73,778 \\
\hline $\mathrm{Al}_{2} \mathrm{O}_{3}$ & 14,188 \\
\hline $\mathrm{K}_{2} \mathrm{O}$ & 7,744 \\
\hline $\mathrm{Fe}_{2} \mathrm{O}_{3}$ & 2,49 \\
\hline $\mathrm{CaO}$ & 0,969 \\
\hline $\mathrm{SO}_{3}$ & 0,435 \\
\hline $\mathrm{Cs}_{2} \mathrm{O}$ & 0,276 \\
\hline $\mathrm{MnO}$ & 0,061 \\
\hline $\mathrm{Rb}_{2} \mathrm{O}$ & 0,037 \\
\hline $\mathrm{ZnO}$ & 0,022 \\
\hline
\end{tabular}

\begin{tabular}{|l|r|}
\hline \multicolumn{2}{|c|}{ Amostra B - Retido $\# 200$} \\
\hline Oxido & Percentual \\
\hline $\mathrm{SiO}_{2}$ & 73,455 \\
\hline $\mathrm{Al}_{2} \mathrm{O}_{3}$ & 16,562 \\
\hline $\mathrm{K}_{2} \mathrm{O}$ & 7,499 \\
\hline $\mathrm{CaO}$ & 1,179 \\
\hline $\mathrm{Fe}_{2} \mathrm{O}_{3}$ & 0,869 \\
\hline $\mathrm{SO}_{3}$ & 0,351 \\
\hline $\mathrm{MnO}_{2}$ & 0,044 \\
\hline $\mathrm{Rb}_{2} \mathrm{O}$ & 0,024 \\
\hline $\mathrm{GeO}_{2}$ & 0,017 \\
\hline
\end{tabular}

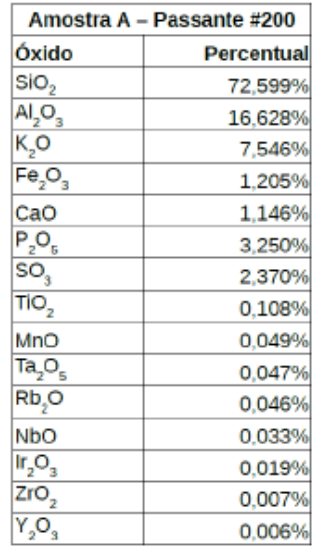

a)

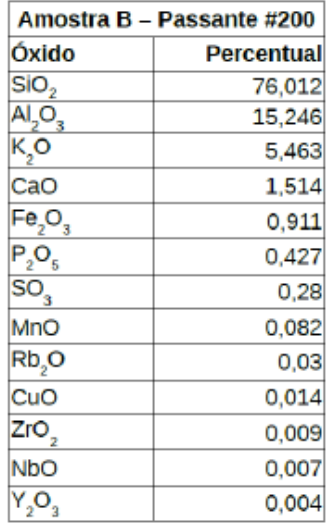

b)

Figura 2: Análises de FRX - a) Retito e passante \#200 amostra A; b) Retido e passante \#200 amostras B.

Para a etapa de corte do maciço rochoso foram identificadas as oportunidades nas boas técnicas operacionais e mudança no processo. Diante desta expectativa, resolveu-se mapear as frentes de lavra da pedreira e determinar as famílias de falhas, foram realizadas 06 medidas de linhas, para fins de determinar os deslocamentos associados com a estrias. Estas estrias foram catalogadas em campo através de medições de azimute e inclinação, com uso de bússola e clinômetro e compilação e tratamento dos dados através do software open stereo, com intuito de se determinar o plano resultante e assim o avanço ideal para minimizar a produção de resíduos nesta etapa da operação de lavra.

Com as medidas das linhas de face foi determinado a direção de mergulho média que foi de $191,3^{\circ}$, e o plano de falha resultante de $187.5 / 44,3 \mathrm{SW}$. Sendo assim, sugere-se que o corte de corrida acompanhe a direção da foliação $\mathrm{N} 44,3 \mathrm{~W}$ para que diminua-se as perdas no corte. $\mathrm{O}$

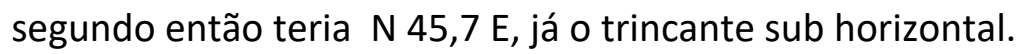

Pode-se ressaltar que o mapeamento de estruturas pode contribuir na melhoria da operação, tornando-a mais segura para a empresa e para o trabalhador, haja vista que identificada a direção de escorregamento de blocos pode-se evitar acidentes de trabalho, sendo assim uma oportunidade de implementação de Produção Mais Lima pela técnica de boas práticas operacionais. 
No tombamento das pranchas, a oportunidade monitorada para aplicação de técnicas de Produção Mais Limpa foi as boas práticas operacionais, além do corte respeitando as estruturas geológicas. É essencial uma boa preparação de uma espécie de colchão de amortecimento para que a prancha caia sobre o piso da praça sem sofrer danos que causem fraturas na rocha. Nesta perspectiva, pode-se reutilizar solos e fragmentos de rochas que estão depositados na pilha de resíduos para servir de colchão

No tocante ao consumo de água, pode-se utilizar também as boas práticas operacionais. De maneira, a sempre está fiscalizando o rendimento do fio e amperagem em que a máquina cortadora está operando, pois é um bom indicativo para o uso moderado da água.

No que diz respeito a embalagens plásticas, o que pode ser sugerido é uma revisão do plano de corte com a argamassa expansiva, visando aumentar o espaçamento entre furos e carregar os furos de maneira alternada, carrega um e deixar o outro vazio. Desta maneira poderá diminuir o consumo de argamassa e consequentemente a geração do resíduo plástico.

Quanto ao consumo de combustíveis e emissões, mais uma vez pode-se elencar as boas práticas operacionais para que possa ser implementada no processo. Realizar paradas para manutenção preditiva e preventiva. Quando o equipamento estiver ocioso desligar sempre que possível o motor para evitar consumo desnecessário. Por fim, os fragmentos de rocha depositados na pilha de resíduo podem ser reciclados internamente, de modo a buscar tornar o resíduo em produto e diminuir geração e acumulação de resíduos.

\section{CONCLUSÕES}

A partir da análise do diagnóstico de resíduos e processo e dos dados, que foram gerados por meio do estudo de caso da Pedreira Granorte, conclui-se que a ferramenta de gestão ambiental Produção Mais Limpa pode ser bastante útil aos mineradores deste setor. Basta citar a situação de resíduos da empresa, em que mais de $99,97 \%$ dos resíduos gerados, relacionados à lavra são de fragmentos de rochas. Percebe-se na literatura que são raros os estudos que visam a implementação de Produção Mais Limpa na lavra de rochas ornamentais, em sua maioria se detém apenas ao beneficiamento deste recurso. As pesquisas demonstram que boa parte das soluções sugeridas por pesquisadores estão ligadas a reciclagem de resíduos do beneficiamento de rochas ornamentais. Desta maneira a Produção Mais Limpa aplicada a operação de lavra pode levar este tipo de mineração a práticas sustentáveis que visem à economia e o uso racional dos recursos naturais, principalmente água, recurso mineral e consumo de combustível.

O município de Parelhas tem se destacado na mineração de rochas ornamentais devido possuir uma boa diversidade de materiais exóticos. Entretanto, o destaque para atividade econômica deve vir atrelado aos cuidados com o meio ambiente, haja vista que estudos demonstram que esta atividade é potencialmente poluidora principalmente por produzir grandes volumes de resíduos sólidos, e neste caso os de rochas.

Para solucionar a geração de resíduos sólidos na atividade de lavra de rocha ornamental, o diagnóstico de resíduos se demonstra essencial para identificar as oportunidades no processo e que 
podem trazer melhorias ambientais na busca pelo desenvolvimento sustentável desta atividade através do uso racional dos recursos naturais.

Combinações de técnicas de Produção Mais Limpa podem corroborar com o meio ambiente, com a preservação do recurso mineral, redução do consumo de combustíveis e consequente diminuição de emissões, além de trazer economia de recursos financeiros para empresa, tendo em vista que pode diminuir o número de paradas por manutenções corretivas, como também o equilíbrio da utilização e disponibilidade destes equipamentos.

No diagnóstico de resíduos sólidos e processos na atividade de lavra, a operação de decapeamento e limpeza teve a maior quantidade de resíduos gerados com $35 \%$ do total, a atividade de corte com $26 \%$, na etapa de tombamento de pranchas, a quantidade de resíduos foi de $21 \%$, estas duas últimas podem estar relacionadas a mudanças de tonalidade do material (estético), microfraturamentos da rocha, direção de avanço e corte. No desdobramento as perdas são da ordem de $15 \%$, e estão relacionadas principalmente ao esquadrejamento que visa deixar os blocos com as faces planas, formando paralelepípedos com padrão de mercado. A perfuração foi a operação no processo de lavra que menos gerou resíduos sólidos do tipo fragmentos de rochas, tendo 3\% do total. A perfuração foi a operação no processo de lavra que menos gerou resíduos sólidos do tipo fragmentos de rochas, tendo3\% do total.

O consumo de água pode ser diminuído por consequência de eficiências e boas práticas no corte da rocha. Assim o consumo relativo tenderá a diminuir com o aumento do rendimento da pedreira, além de medidas que visem racionalizar o uso do recurso hídrico.

Com foco de redução na fonte da geração de resíduos que é a principal meta de um Planejamento para implementação de Produção Mais Limpa, o mapeamento das estruturas geológicas é uma técnica que combina mudança no processo com as boas práticas operacionais. Estima-se uma redução otimista da ordem de $20 \%$ dos resíduos sólidos gerados, isto pode ser possível com a mudança no avanço da lavra e dos sentidos e direção dos cortes conforme análise apresentada e aprofundamento dos estudos de geologia estrutural e de mecânica de rochas.

Explicitou-se, então, uma metodologia de diagnóstico ambiental, que pode servir de subsídio para a elaboração políticas públicas, que possam vir a viabilizar um pacto pela busca do desenvolvimento sustentável na mineração de rocha ornamental, entre empresa, comunidade e poder público, a partir do monitoramento das operações de lavra e da utilização de indicadores socioambientais.

A análise qualitativa e quantitativa detalhada dos resíduos deve ser feita durante períodos de tempo relativamente longos de aproximadamente 12 meses. A elaboração de um diagnóstico para identificação e caracterização das empresas de extração de rocha ornamental em funcionamento no município de Parelhas-RN, pode promover uma parceria público-privada para criação de um Arranjo Produtivo Local - APL no município e circunvizinhança. 


\section{REFERÊNCIAS}

BRASIL (2017). Ministério da Indústria, Comércio Exterior e Serviços - MDIC (2017). Dados de exportação do município de Parelhas-RN. 2017. Disponível em: $<$ http://www.mdic.gov.br/comercio-exterior/estatisticas-de-comercio-exterior/comex-vis/framemunicipio?municipio=2408904>. Acesso em: 14 jun.

CAMPOS et al (2009). Tratamento e aproveitamento de resíduos de rochas ornamentais e de revestimento, visando mitigação de impacto ambiental. XXIII Simpósio Geologia do Nordeste; VII Simpósio de Rochas Ornamentais do Nordeste. In: SIMPÓSIO DE ROCHAS ORNAMENTAIS DO NORDESTE, VII, Fortaleza.

CURI, Adilson (2014). Minas a céu aberto: planejamento de lavra. São Paulo: Oficina de Textos, 2014.

MENEZES, R.G. (2005). Rochas Ornamentais e de Revestimento: Conceitos, Tipos e Caracterização Tecnológica. In: Universidade Federal do Rio de Janeiro - UFRJ, em associação com o Centro de Tecnologia Mineral - CETEM - do Ministério de Ciência e Tecnologia e Centro Tecnológico de Mármore e Granito - CETEMAG (ES).

SAMPIERI, Roberto Hernández; COLLADO, Carlos Fernández; LUCIO, Pilar Baptista (2006). Metodologia de Pesquisa. São Paulo: McGraw-Hill.

SENAI-RS. SERVIÇO NACIONAL DE APRENDIZAGEM INDUSTRIAL DO RIO GRANDE DO SUL (2003a). Implementação de programas de produção mais limpa. Porto Alegre: CNTL, SERVIÇO NACIONAL DE APRENDIZAGEM INDUSTRIAL.

VENTURI, L. A. B. (2006). Recurso natural: a construção de um conceito. GEOUSP- Espaço e Tempo. $n^{\circ} 20$, p. 9-17, São Paulo, ago. 